\title{
Acute hydrogen-rich water ingestion stimulates cardiac autonomic activity in healthy females
}

\author{
Michal Botek ${ }^{1}$, Barbora Sládečková ${ }^{\bowtie}$, Jakub Krejčí ${ }^{1}$, František Pluháček², and Eliška Najmanová ${ }^{2}$ \\ ${ }^{1}$ Department of Natural Sciences in Kinanthropology, Faculty of Physical Culture, Palacký University Olomouc, Olomouc, Czech \\ Republic; and ${ }^{2}$ Department of Optics, Faculty of Science, Palacký University Olomouc, Olomouc, Czech Republic
}

\begin{abstract}
Background: Hydrogen-rich water (HRW) has been shown to have a stimulating effect on the human body. Objective: The aim of the study was to assess the influence of acute HRW intake on autonomic cardiac regulation during 50 min of rest sitting. Methods: Fourteen healthy females (age $21.7 \pm 1.2$ years, body mass $67.8 \pm 8.7 \mathrm{~kg}$, height $167 \pm 5.5 \mathrm{~cm}$ ) took part in this double-blind, randomized, placebo-controlled trial with crossover design. Heart rate variability (HRV) was monitored in the sitting position after administration of $1260 \mathrm{ml}$ of HRW or placebo. Time domain indexes of HRV as the square root of the mean of the squares of differences between adjacent RR intervals (RMSSD), the standard deviation of all RR intervals (SDNN) and the ratio of SDNN/RMSSD as an index of sympatho-vagal balance were used to assess autonomic cardiac response. The values were transformed using natural logarithm (Ln). Results: After administration of HRW, we found significantly increased ratio Ln SDNN/RMSSD when comparing it to placebo in $25 \mathrm{~min}$ (HRW: $0.40 \pm 0.30$, placebo: $0.26 \pm 0.25, p=.049$ ) and $35 \min$ (HRW: $0.44 \pm 0.30$, placebo: $0.28 \pm 0.28, p=.029$ ) of rest sitting. Ln SDNN was significantly increased after HRW administration when compared to placebo in $45 \mathrm{~min}$ (HRW: $4.41 \pm 0.42 \mathrm{~ms}$, placebo: $4.28 \pm 0.31 \mathrm{~ms}, p=.049$ ) of rest sitting. Conclusions: Acute HRW ingestion induced a relative increase in sympathetic activity between 25 and 35 min post-ingestion, whereas vagal activity was not affected.
\end{abstract}

Keywords: molecular hydrogen, autonomic nervous system, heart rate variability, sympathetic activity, time domain indexes

\section{Introduction}

Molecular hydrogen $\left(\mathrm{H}_{2}\right)$ has been described as an antiinflammation agent (Ohta, 2015). Moreover, $\mathrm{H}_{2}$ was reported as a strong selective antioxidant itself or gas able to improve the activity of the antioxidant system showing cytoprotective effect (Ohsawa et al., 2007). The antioxidative effect of $\mathrm{H}_{2}$ was proved by neutralization of reactive oxidative species and reactive nitrogen species (Nicolson et al., 2016). The higher level of reactive oxidative and nitrogen species is associated with aging, daily stress, demanding physical activity etc., where stressors can increase sympathetic activity, and consequently the oxidative stress (Steptoe et al., 2007). The human body could be exposed to $\mathrm{H}_{2}$ in several ways as follows, inhalation of $\mathrm{H}_{2}$ (Fukuda et al., 2007), intravenous infusion (Ishibashi et al., 2014) of $\mathrm{H}_{2}$ or by more practical methods as intake of hydrogen rich water (HRW; Kajiyama et al., 2008) and $\mathrm{H}_{2}$ bathing (Kawamura et al., 2016). HRW is considered to be the easiest and the safest method of delivering $\mathrm{H}_{2}$ to the human body (Nicolson et al., 2016). Clinical and animal studies have demonstrated that HRW intake is linked to numerous health benefits, such as improvement in lipid and glucose metabolism in diabetes patients (Kajiyama et al., 2008), quality of life after radiotherapy treatment in regards to reducing the biological reaction to radiation-induced oxidative stress (Kang et al., 2011), other studies showed a reduction of mitochondrial DNA damage (Tomofuji et al., 2014) and improved autonomic cardiac function after 4 weeks of administration in healthy adults (Mizuno et al., 2017). In addition to clinical studies, there is positive effect also in physically active humans while HRW was reported to reduce blood acidosis (Ostojic \& Stojanovic, 2014) and lactate concentration (Aoki et al., 2012; Botek et al., 2019, 2021), improve perceptual and ventilatory response to exercise (Botek et al., 2019) or having an antifatigue effect (Aoki et al., 2012; Botek et al., 2020, 2021; Da Ponte et al., 2018). Zanini et al. (2020) found an alternative effect of HRW to caffeine in terms of sleep deprivation where HRW was suggested to have a stimulating effect on the brain, particularly improved sensory stimulation.

Autonomic nervous system (ANS) activity is commonly noninvasively assessed by heart rate variability (HRV; Malik, 1997; Task Force of the European Society of Cardiology, 1996). HRV reflects the complexity of the physiological system and mirrors the time variations in the subsequent heartbeats intervals which is primarily associated with respiratory related vagal activity (Acharya et al., 2006). It has been reported that HRV analysis sensitively

\footnotetext{
$\triangle$ Corresponding author: Barbora Sládečková, e-mail barbora.sladeckova@upol.cz, ORCID ${ }^{\circledR}$ record https://orcid.org/0000-0001-6862-4571 Article history: Received November 25 2020, Accepted April 6 2021, Published April 272021

Copyright: (c) 2021 The Author(s). Published by Palacký University Olomouc. This is an open access article distributed under the terms of the Creative Commons Attribution License (https://creativecommons.org/licenses/by/4.0/), which permits unrestricted use, distribution, and reproduction in any medium, provided the original author and source are credited. This license does not cover any third-party material that may appear with permission in the article.
} 
identifies even the tiny changes in autonomic cardiac alterations that may occur with acute intake dose of water (Chen et al., 2004).

A previous study has shown that $7.5 \mathrm{ml} \cdot \mathrm{kg}^{-1}$ of water intake elicited many cardiovascular changes, such as total peripheral resistance, HRV and cardiovagal baroreflex sensitivity, decrease in heart rate (HR), and a parallel increase in HF component showing enhanced vagal activity (Brown et al., 2005). Water drinking activates both the sympathetic (Scott et al., 2001) and vagal branches (Routledge et al., 2002) of the ANS. Physiological response to water drinking in healthy subjects may be an integrated response including increased sympathetic vasoconstrictor activity and at the same time parallel increase of the vagal activity that prevents a rise in arterial pressure by reducing cardiac output (Jordan et al., 2000). This result corresponds to the study of Chen et al. (2018) who found a rise in cardiac vagal activity at 15 min post water intake. Similarly, Peçanha et al. (2014) reporting faster cardiac vagal reactivation due to water intake after high intensity exercise.

The present study aimed to investigate the effects of acute HRW ingestion on autonomic cardiac response in young healthy females during sitting at rest. In this regard, we hypothesized that there would be a significant increase in sympathetic activity and concurrent reduction in vagal activity.

\section{Methods}

\section{Participants}

Fourteen healthy female university students (age $21.7 \pm 1.2$ years, body mass $67.8 \pm 8.7 \mathrm{~kg}$, height $167 \pm 6 \mathrm{~cm}$ ) took part in this study. Prior to testing, all tested participants were informed about the testing procedures and the aim of the research. The day before testing, participants were not exposed to any exhaustive activity. The participants were advised to avoid drinking coffee, tea or any other substance potentially affecting the ANS activity, for at least two hours before the testing. The research was conducted in accordance with the Declaration of Helsinki and was approved by the Ethics Committee of the Faculty of Physical Culture, Palacký University Olomouc under reference number $75 / 2017$. The participation of subjects in this research was voluntary and all participants signed informed consent with the testing procedure.

\section{Anthropometric measures}

The participants had their body height $(\mathrm{cm})$ and body mass (kg) measured using the SOEHNLE 7307 (Leifheit, Nassau, Germany).

\section{Experimental protocol}

This is a double-blind, randomized, placebo-controlled trial with a crossover design. The study protocol consisted of 2 laboratory visits (Figure 1). The first visit included a detailed explanation of the course of the experiment, the signing of informed consent, body mass measurement, and the random division of participants into two groups. Each group contained 7 participants. Randomization was performed by means of lots, using an equal number of strips with two color (red and blue). Participants drew only one strip whilst blinded. Then the experiment itself began.

Data for HRV analysis were measured in five segments (Figure 1). Each segment lasted $5 \mathrm{~min}$, as recommended for standardized short-term recording (Task Force of the

Figure 1 Overview of the study protocol

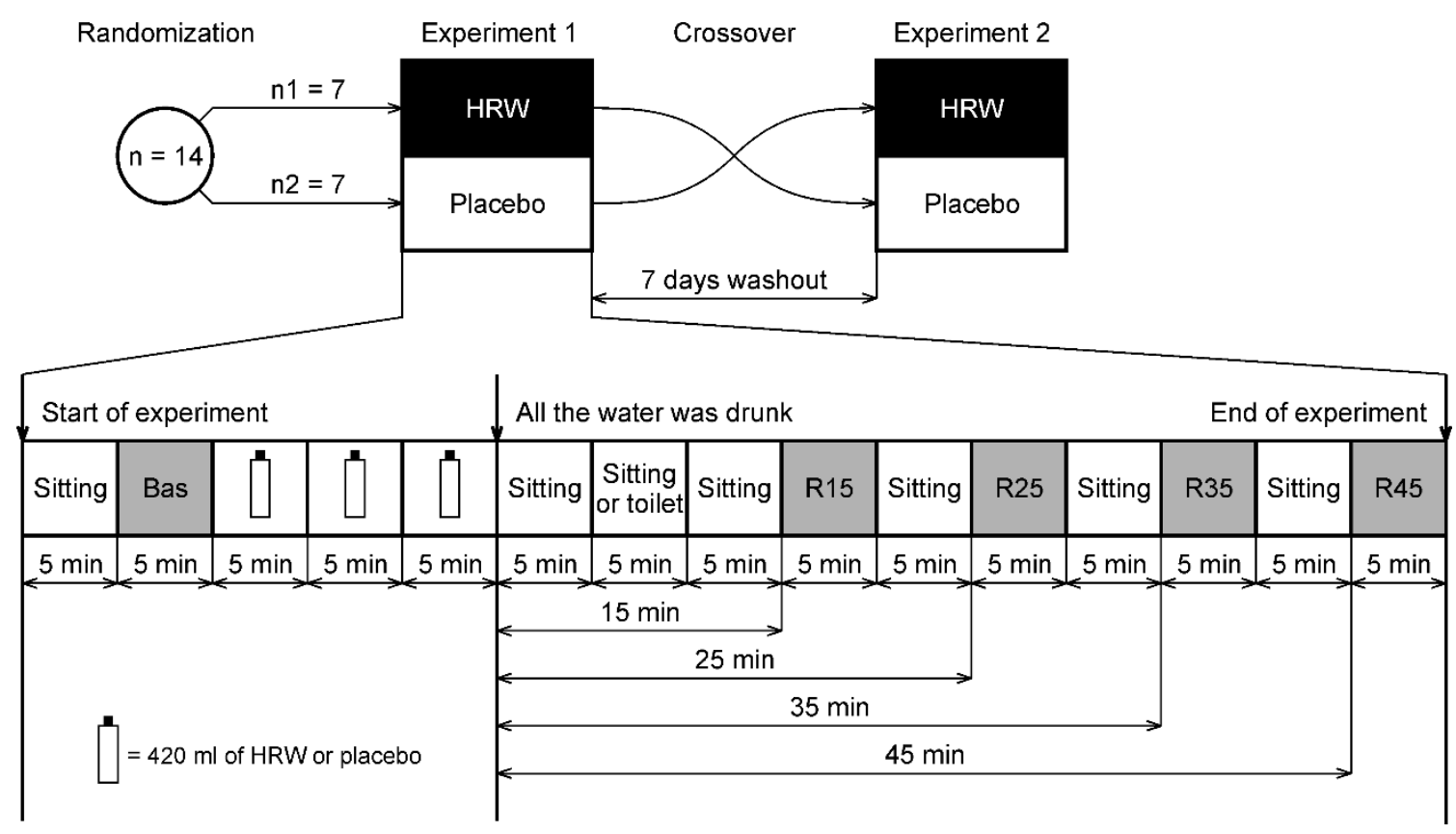

Note. RR intervals were recorded in grey coloured segments. HRW = hydrogen rich water; Bas = baseline segment before any water administration; R15, $R 25, R 35$, and $R 45=$ recovery segments $15,25,35$, and 45 min respectively after drinking all the water. 
European Society of Cardiology, 1996). The baseline segment was measured before the administration of any water and was used to calculate HRV reference values. Before the baseline segment, there was a 5 min pause during which the participants sat in a comfortable chair. This pause served as a stabilization period to ensure RR intervals to achieve stationarity.

After completing the measurement of the baseline segment, participants were asked to drink three packages of beverage (HRW or placebo) within a 15 min time frame. It was requested to drink one package every $5 \mathrm{~min}$. After $15 \mathrm{~min}$ and drinking all the water, the recovery time began to count. During the first $10 \mathrm{~min}$, participants were sitting and had the opportunity to visit the toilet to empty their urinary bladder. This was followed by another 5 min stabilization pause in which participants were sitting to achieve stationary RR intervals. 15 min after drinking the last package, the recording of the first recovery segment labelled R15 was started. Participants were asked to sit without excessive movement until the end of the entire experimental protocol, during which three more recovery segments were recorded. The segments started 25, 35, and 45 min after drinking the last package and were labeled R25, R35, and R45 respectively.

After 7 days of washout, a second visit took place, in which beverages were exchanged (HRW for placebo and vice versa).

\section{HRW and placebo preparation}

A total volume of $1260 \mathrm{ml} \mathrm{HRW} \mathrm{(Aquastamina} \mathrm{HRW,}$ Nutristamina, Ostrava, Czech Republic) or placebo (Aquastamina $\mathrm{H}_{2}$ free, Nutristamina, Ostrava, Czech Republic) was administrated in three doses ( $420 \mathrm{ml}$ each) within $15 \mathrm{~min}$. HRW and a placebo was served in identical packages so participants were not able to distinguish between HRW and placebo because $\mathrm{H}_{2}$ is colorless, odorless and tasteless. HRW was produced by infusing $\mathrm{H}_{2}$ under high pressure directly into water. The characteristic of both HRW and placebo (Table 1) were determined using the $\mathrm{pH} / \mathrm{ORP} /$ Temperature-meter (AD14, Adwa Instruments, Szeged, Hungary) and H2Blue reagent (H2 Sciences, Henderson, NV, USA) according to the manufacturer instructions.

\section{HRV monitoring}

To calculate $H R$ and HRV variables, RR intervals were recorded using a Polar HR monitor (V800, Polar, Kempele, Finland) with a resolution of $1 \mathrm{~ms}$. All participants

Table 1 Chemical characteristic of hydrogen rich water and placebo

\begin{tabular}{lcc}
\hline Property & HRW & Placebo \\
\hline $\mathrm{pH}$ & 7.8 & 7.6 \\
$\mathrm{ORP}(\mathrm{mV})$ & -652 & +170 \\
Temperature $\left({ }^{\circ} \mathrm{C}\right)$ & 22 & 22 \\
$\mathrm{H}_{2}$ concentration $(\mathrm{ppm})$ & 0.9 & 0.0 \\
\hline
\end{tabular}

Note. $\mathrm{HRW}=$ hydrogen rich water; $\mathrm{ORP}=$ oxidation reduction potential. wore watches and chest straps. Participants were allowed to breathe spontaneously during the RR recording. Paced breathing was not used because voluntary control of breathing has been shown to affect HRV indexes (Patwardhan et al., 1995). However, time domain analysis of HRV is thought to be resistant to the effects of breathing frequency compared to spectral analysis (Penttilä et al., 2001). RR intervals were transferred to a computer using the Polar Flow cloud service. Artifacts (ectopic beats, missing beats, etc.) were identified by visual inspection and simply deleted because the deletion method provided the best overall performance (Lippman et al., 1994). The square root of the mean of the squares of differences between adjacent RR intervals (RMSSD), the standard deviation of all RR intervals (SDNN), and the SDNN to RMSSD ratio (SDNN/RMSSD) were calculated using a custom application written in MATLAB (Version 8.4; MathWorks, Natick, MA, USA) according to the formulas published by Wang and Huang (2012). RMSSD is assumed to be an index of vagal activity (Task Force of the European Society of Cardiology, 1996), SDNN is assumed to be an index of total variability (Aubert et al., 2003; Malik, 1997), and SDNN/ RMSSD is considered as an index of sympatho-vagal balance (Wang \& Huang, 2012). Because raw HRV indexes were not normally distributed, they were transformed using natural logarithm $(\mathrm{Ln})$.

\section{Statistical analysis}

MATLAB v8.4 with Statistics Toolbox v9.1 (MathWorks, Natick, MA, USA) was used for statistical analyses. The Kolmogorov-Smirnov test was used to verify that the studied variables have a normal distribution. The normal distribution was not rejected for all dependent variables (HR: $p=.23$, Ln RMSSD: $p=.36$, Ln SDNN: $p=.081$, Ln SDNN/RMSSD: $p=.38$ ) and therefore the use of statistical methods assuming a normal distribution was appropriate. A linear model with one random factor (participants), two fixed factors (beverage and time), and interaction (beverage $\times$ time) was used to evaluate the effects of HRW and time on the dependent variables. The beverage factor was void in the baseline segment because neither HRW nor placebo was administered before the baseline segment. Fisher's LSD post-hoc test was used to calculate the significance of the difference between the two selected means. For all tests, $p<.05$ was considered statistically significant.

Arithmetic mean and standard deviation were used as descriptive statistics. The partial eta-squared $\left(\eta_{p}^{2}\right)$ was used as an effect size for factors of the linear model and Cohen's $d$ was used as an effect size for pairwise comparisons. The magnitude of the effect size was interpreted according following thresholds (Cohen, 1988): trivial $\left(\eta_{\mathrm{p}}^{2}<.01\right.$, $d<0.2)$, small $\left(\eta_{\mathrm{p}}^{2} \geq .01, d \geq 0.2\right)$, medium $\left(\eta_{\mathrm{p}}^{2} \geq .06\right.$, $d \geq 0.6)$, large $\left(\eta_{\mathrm{p}}^{2} \geq .14, d \geq 1.2\right)$.

\section{Results}

A statistically significant beverage factor was found for HR and Ln SDNN/RMSSD with medium and small effect size, respectively (Table 2). A statistically significant time 
factor was found for all dependent variables and the effect size ranged from small to large (Table 2). The interaction between beverage and time factor was not statistically significant for any dependent variable (Table 2).

Pairwise comparisons are displayed in Figure 2. For $\mathrm{HR}$, pairwise comparisons showed significant differences between HRW and placebo at R25 (HRW: $70.1 \pm 11.5$ beats $\cdot \mathrm{min}^{-1}$, placebo: $66.9 \pm 10.1$ beats $\cdot \mathrm{min}^{-1}, p=.033$, $d=0.58$, small effect) and R35 (HRW: $71.3 \pm 9.9$ beats $\cdot \mathrm{min}^{-1}$, placebo: $67.9 \pm 9.5$ beats $\cdot \mathrm{min}^{-1}, p=.021$, $d=0.62$, medium effect). HR at all recovery times after both HRW and placebo administration was significantly reduced compared to baseline $(p \leq .001, d$ ranging from 0.89 to 1.64 , medium to large effects).
For Ln RMSSD, no statistically significant pairwise comparison between HRW and placebo was found ( $p \geq .20, d$ ranging from -0.35 to 0.11 , trivial to small effects). Ln RMSSD at all recovery times after both HRW and placebo administration was significantly elevated compared to baseline ( $p \leq .004, d$ ranging from -1.57 to -0.80 , medium to large effects).

For Ln SDNN, a significant difference between HRW and placebo was found in R45 ( $p=.049, d=0.53$, small effect). Ln SDNN at all recovery times after HRW administration was significantly elevated compared to baseline ( $p \leq .001, d$ ranging from -1.16 to -0.90 , large effects). However, Ln SDNN after placebo administration was significantly elevated only at R15, R25, and R35 ( $p \leq .020$, $d$

Table 2 Results of statistical analysis using a linear mixed-effects model

\begin{tabular}{|c|c|c|c|c|c|c|}
\hline Variable & \multicolumn{2}{|c|}{ Beverage factor } & \multicolumn{2}{|c|}{ Time factor } & \multicolumn{2}{|c|}{ Interaction } \\
\hline $\mathrm{HR}$ (beats $\cdot \mathrm{min}^{-1}$ ) & .002 & .075 & $<.001$ & .403 & .50 & .020 \\
\hline Ln RMSSD (ms) & .29 & .009 & $<.001$ & .385 & .64 & .014 \\
\hline Ln SDNN (ms) & .14 & .019 & $<.001$ & .303 & .28 & .032 \\
\hline Ln SDNN/RMSSD & .009 & .057 & .014 & .100 & .31 & .030 \\
\hline
\end{tabular}

Note. $\mathrm{HR}=$ heart rate; $\mathrm{Ln}=$ natural logarithm; RMSSD = square root of the mean of the squares of differences between adjacent RR intervals; SDNN = standard deviation of all RR intervals; SDNN/RMSSD = SDNN to RMSSD ratio.

Figure 2 Effect of hydrogen rich water (full circle) compared to placebo (empty circle) on heart rate (HR) and heart rate variability variables
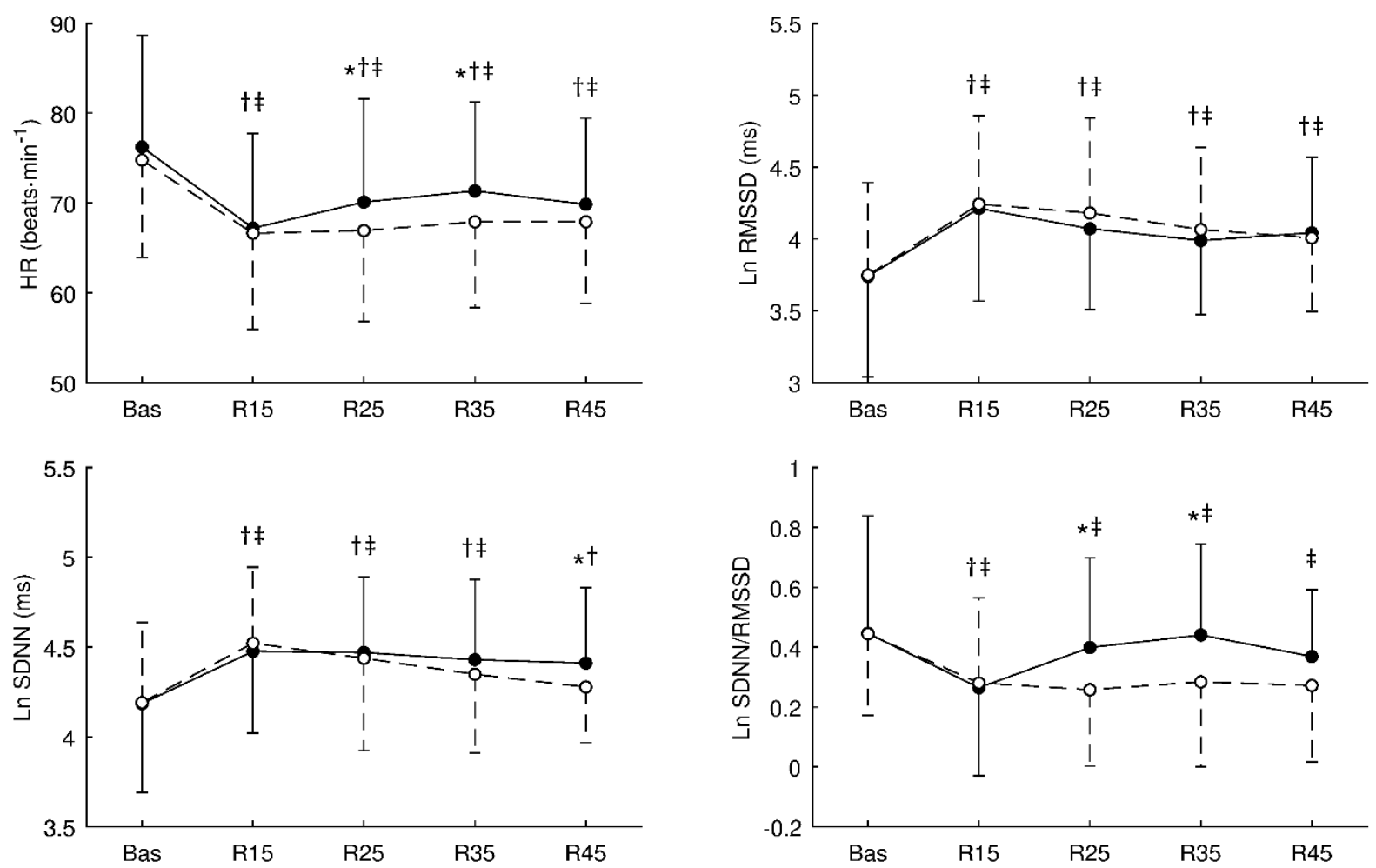

Note. Values are presented as the mean and standard deviation. Ln = natural logarithm; RMSSD = square root of the mean of the squares of differences between adjacent RR intervals; SDNN = standard deviation of all RR intervals; SDNN/RMSSD = SDNN to RMSSD ratio; Bas = baseline segment before any water administration; R15, R25, R35, and R45 = recovery segments $15,25,35$, and 45 min respectively after drinking all the water.

*statistically significant $(p<.05)$ difference between hydrogen rich water and placebo at the same time; tstatistically significant $(p<.05)$ difference between this time and the baseline when hydrogen rich water was administered; łstatistically significant $(p<.05)$ difference between this time and the baseline when placebo was administered. 
ranging from -1.32 to -0.63 , large effects). The difference at R45 was not statistically significant $(p=.20, d=-0.34$, small effect).

For Ln SDNN/RMSSD, pairwise comparisons showed significant differences between HRW and placebo at R25 ( $p=.049, d=0.53$, small effect) and R35 ( $p=.029$, $d=0.59$, small effect). Ln SDNN/RMSSD after HRW administration was significantly reduced at $\mathrm{R} 15$ ( $p=.012$, $d=0.69$, medium effect) and returned at R25, R35, and R45 to values not significantly different from baseline ( $p \geq .28$, $d$ ranging from 0.02 to 0.29 , trivial to small effects). However, Ln SDNN/RMSSD after placebo administration remained significantly reduced at all recovery times ( $p \leq .026, d$ ranging from 0.60 to 0.70 , medium effects).

\section{Discussion}

The aim of this study was to assess the influence of acute HRW intake on ANS activity response for the following $50 \mathrm{~min}$ in rest sitting. The main findings of the present study revealed that $1260 \mathrm{ml}$ of HRW compared to placebo significantly modified autonomic cardiac regulation in healthy females.

Specifically, in R15 of recovery after ingestion of 1260 $\mathrm{ml} \mathrm{HRW}$ and/or placebo we observed a significant increase in vagal activity with the concomitant reduction in $\mathrm{HR}$ compared to baseline. A similar effect of acute water ingest was reported also by Chen et al. (2004) whose results, taken in a lying position revealed increased cardiac vagal activity after $500 \mathrm{ml}$ of water administration. A significant rise in HRV indicating a shift towards increased vagal activity accompanied by reduced HR from $67.6 \pm 2.0$ to $60.7 \pm 2.4$ beats. $\mathrm{min}^{-1}$ was also found by Routledge et al. (2002) after $500 \mathrm{ml}$ of water administration. Data in mentioned study were measured in a supine position and reduction in HR peaked between 20 and $25 \mathrm{~min}$. In another study, Brown et al. (2005) reported that $7.5 \mathrm{ml} \cdot \mathrm{kg}^{-1}$ of water intake induced an immediate increase in cardiac vagal activity and its maintenance for at least $30 \mathrm{~min}$ as was found in our study. The increase in vagal activity and concomitant decrease of HR may be the underlying mechanism of reducing high blood pressure which commonly occurs after water administration (Callegaro et al., 2007).

In contrast, in previous studies neither $480 \mathrm{ml}$ (Jordan et al., 2000) nor $500 \mathrm{ml}$ (Schroeder et al., 2002) water drinking in young healthy people did not cause changes in blood pressure, and the authors concluded that water drinking in healthy subjects may be an integrated response consisting of increased sympathetic vasoconstrictor activity and a parallel increase in cardiac vagal activity. Thus, the increase in peripheral resistance is counteracted by a fall in cardiac output. Increased sympathetic activity after water drinking confirmed Scott et al. (2001) who examined muscle sympathetic neural activity, leading to peripheral vasoconstriction. In our study, significantly reduced HR compared to baseline value was found peaking at $35 \mathrm{~min}$ after administration with a significant difference between HRW and placebo. We assume that HR was reduced by the influence of the same dose of water, however, the decrease in HR after HRW administration was smaller compared to placebo, likely due to the stimulating effect of HRW.

We found a significant shift in sympathovagal balance towards the sympathetic side 25 and $35 \mathrm{~min}$ after HRW administration, whereas the vagal activity was not significantly affected by HRW administration. We, therefore, assume that the increase in HR was caused by function changes in sympathetic activity. Interestingly, a time-frame of the relative increase in sympathetic activity after HRW ingestion that was detected in our study, noticeably matched with dynamics of the $\mathrm{H}_{2}$ peak in the breath in humans (Shimouchi et al., 2009). We explain the loss of significant differences in HR and Ln SDNN/RMSSD beyond $35 \mathrm{~min}$ by the utilizing of $\mathrm{H}_{2}$ in the human body or by exhaling of $\mathrm{H}_{2}$ from the body. The stimulating effect of HRW on brain circuits was recently found also by Zanini et al. (2020) who examined markers of alertness in sleep deprived men and women. Mentioned authors reported a similar effect of HRW compared to caffeine when HRW positively affected orienting to sensory stimulation and concluded that HRW might be advanced as a safe and effective alternative to caffeine. In contrast to our results, Mizuno et al. (2017) reported an attenuation in sympathetic activity (low frequency) during the resting state in adult volunteers after 4-week administration of $600 \mathrm{ml} \mathrm{HRW}$ per day and concluded that besides autonomic function, HRW intake may increase central nervous system functions improving mood and anxiety. In regard to ANS activity, discrepancies between our results and the study mentioned above might be explained by a different period of HRW administration. From a practical point of view, we feel that if acute $\mathrm{H}_{2}$ administration may stimulate the sympathetic cardiac system, therefore it would not be recommended to ingest HRW at least $60 \mathrm{~min}$ before sleeping in order to avoid issues with falling asleep.

One limitation of this study might be the lack of information about blood pressure response to pre and post HRW administration. Another limit is that we used a constant amount of HRW per subject and we did not adjust it to the body mass of subjects. The menstrual cycle was not evaluated in this study, which can be considered as another limit as well as the low sample size.

\section{Conclusions}

Acute ingestion of $1260 \mathrm{ml}$ of HRW in healthy females induced a relative increase in sympathetic cardiac activity between 25 and 35 min post-ingestion, whereas vagal activity was not affected by $\mathrm{H}_{2}$ administration.

\section{Acknowledgments}

This study was supported by Palacký University Olomouc (grant number IGA_FTK_2020_007). The authors would like to thank Hana Baleková for collecting data for this study.

\section{Conflict of interest}

The authors report no conflict of interest. 


\section{References}

Acharya, U. R., Joseph, K. P., Kannathal, N., Lim, C. M., \& Suri, J. S. (2006). Heart rate variability: A review. Medical and Biological Engineering and Computing, 44(12), 1031-1051. https://doi.org/10.1007/s11517-006-0119-0

Aoki, K., Nakao, A., Adachi, T., Matsui, Y., \& Miyakawa, S. (2012). Pilot study: Effects of drinking hydrogen-rich water on muscle fatigue caused by acute exercise in elite athletes. Medical Gas Research, 2(1), Article 12. https://doi. org/10.1186/2045-9912-2-12

Aubert, A. E., Seps, B., \& Beckers, F. (2003). Heart rate variability in athletes. Sports Medicine, 33(12), 889-919. https://doi.org/10.2165/00007256-200333120-00003

Botek, M., Krejčí, J., McKune, A. J., \& Sládečková, B. (2020). Hydrogen-rich water supplementation and up-hill running performance: Effect of athlete performance level. International Journal of Sports Physiology and Performance, 15(8), 1193-1196. https://doi.org/10.1123/ijspp.2019-0507

Botek, M., Krejčí, J., McKune, A. J., Sládečková, B., \& Naumovski, N. (2019). Hydrogen rich water improved ventilatory, perceptual and lactate responses to exercise. International Journal of Sports Medicine, 40(14), 879-885. https:// doi.org/10.1055/a-0991-0268

Botek, M., Krejčí, J., McKune, A., Valenta, M., \& Sládečková, B. (2021). Hydrogen rich water consumption positively affects muscle performance, lactate response, and alleviates delayed onset of muscle soreness after resistance training. Journal of Strength and Conditioning Research. Advance online publication. https://doi.org/10.1519/JSC.0000000000003979

Brown, C. M., Barberini, L., Dulloo, A. G., \& Montani, J. P. (2005). Cardiovascular responses to water drinking: Does osmolality play a role? American Journa of Physiology - Regulatory, Integrative and Comparative Physiology, 289(6), R1687-R1692. https://doi.org/10.1152/ajpregu.00205.2005

Callegaro, C. C., Moraes, R. S., Negrao, C. E., Trombetta, I. C., Rondon, M. U., Teixeira, M. S., Silva, S. C., Ferlin, E. L., Krieger, E. M., \& Ribeiro, J. P. (2007). Acute water ingestion increases arterial blood pressure in hypertensive and normotensive subjects. Journal of Human Hypertension, 21(7), 564-570. https://doi. org $/ 10.1038 /$ s.j.hh. 1002188

Chen, C. L., Lin, H. H., Orr, W. C., Yang, C. C., \& Kuo, T. B. (2004). Transfer function analysis of heart rate variability in response to water intake: Correlation with gastric myoelectrical activity. Journal of Applied Physiology, 96(6), 2226-2230. https://doi.org/10.1152/japplphysiol.01037.2003

Chen, W., Chen, L., Chen, Z., Xiang, Y., Liu, S., Zhang, H., \& Wang, J. (2018). Influence of the water-drinking test on intraocular pressure, Schlemm's canal, and autonomic nervous system activity. Investigative Ophthalmology \& Visual Science, 59(8), 3232-3238. https://doi.org/10.1167/iovs.18-23909

Cohen, J. (1988). Statistical power analysis for the behavioral sciences (2nd ed.). Routledge. https://doi.org/10.4324/9780203771587

Da Ponte, A., Giovanelli, N., Nigris, D., \& Lazzer, S. (2018). Effects of hydrogen rich water on prolonged intermittent exercise. Journal of Sports Medicine and Physical Fitness, 58(5), 612-621. https://doi.org/10.23736/S0022-4707.17.06883-9

Fukuda, K. I., Asoh, S., Ishikawa, M., Yamamoto, Y., Ohsawa, I., \& Ohta, S. (2007). Inhalation of hydrogen gas suppresses hepatic injury caused by ischemia/reperfusion through reducing oxidative stress. Biochemical and Biophysical Research Communications, 361(3), 670-674. https://doi.org/10.1016/i.bbrc.2007.07.088

Ishibashi, T., Sato, B., Shibata, S., Sakai, T., Hara, Y., Naritomi, Y., Koyanagi, S. Hara, H., \& Nagao, T. (2014). Therapeutic efficacy of infused molecular hydrogen in saline on rheumatoid arthritis: A randomized, double-blind, placebocontrolled pilot study. International Immunopharmacology, 21(2), 468-473. https://doi.org/10.1016/i.intimp.2014.06.001

Jordan, J., Shannon, J. R., Black, B. K., Ali, Y., Farley, M., Costa, F., Diedrich, A. Robertson, R. M., \& Robertson, D. (2000). The pressor response to water drinking in humans: A sympathetic reflex? Circulation, 101(5), 504-509. https://doi. org/10.1161/01.cir.101.5.504

Kajiyama, S., Hasegawa, G., Asano, M., Hosoda, H., Fukui, M., Nakamura, N., Kit awaki, J., Imai, S., Nakano, K., Ohta, M., Adachi, T., Obayashi, H., \& Yoshikawa, T. (2008). Supplementation of hydrogen-rich water improves lipid and glucose metabolism in patients with type 2 diabetes or impaired glucose tolerance. Nutrition Research, 28(3), 137-143. https://doi.org/10.1016/j.nutres.2008.01.008

Kang, K. M., Kang, Y. N., Choi, I. B., Gu, Y., Kawamura, T., Toyoda, Y., \& Nakao, A. (2011). Effects of drinking hydrogen-rich water on the quality of life of patients treated with radiotherapy for liver tumors. Medical Gas Research, 1(1), Article 11. https://doi.org/10.1186/2045-9912-1-11

Kawamura, T, Gando, Y, Takahashi, M., Hara, R., Suzuki, K., \& Muraoka, I. (2016). Effects of hydrogen bathing on exercise-induced oxidative stress and delayed-onset muscle soreness. Japanese Journal of Physical Fitness and Sports Medicine, 65(3), 297-305. https://doi.org/10.7600/jspfsm.65.297

Lippman, N., Stein, K. M., \& Lerman, B. B. (1994). Comparison of methods for removal of ectopy in measurement of heart rate variability. American Journal of Physiology - Heart and Circulatory Physiology, 267(1), H411-H418. https:// doi.org/10.1152/aipheart.1994.267.1.h411

Malik, M. (1997). Time-domain measurement of heart rate variability. Cardiac Electrophysiology Review, 1(3), 329-334. https://doi.org/10.1023/A:1009912905325

Mizuno, K., Sasaki, A. T., Ebisu, K., Tajima, K., Kajimoto, O., Nojima, J., Kuratsune, H., Hori, H., \& Watanabe, Y. (2017). Hydrogen-rich water for improvements of mood, anxiety, and autonomic nerve function in daily life. Medical Gas Research, 7(4), 247-255. https://doi.org/10.4103/2045-9912.222448

Nicolson, G. L., de Mattos, G. F., Settineri, R., Costa, C., Ellithorpe, R., Rosenblatt, S., La Valle, J., Jimenez, A., \& Ohta, S. (2016). Clinical effects of hydrogen administration: From animal and human diseases to exercise medicine. International Journal of Clinical Medicine, 7(1), 32. https://doi.org/10.4236/ijcm.2016.71005

Ohsawa, I., Ishikawa, M., Takahashi, K., Watanabe, M., Nishimaki, K., Yamagata, K., Katsura, K.-I., Katayama, Y., Asoh, S., \& Ohta, S. (2007). Hydrogen acts as a therapeutic antioxidant by selectively reducing cytotoxic oxygen radicals. Nature Medicine, 13(6), 688-694. https://doi.org/10.1038/nm1577

Ohta, S. (2015). Molecular hydrogen as a novel antioxidant: Overview of the advantages of hydrogen for medical applications. In E. Cadenas \& L. Packer (Eds.), Methods in enzymology (Vol. 555, pp. 289-317). Academic Press. https:// doi.org/10.1016/bs.mie.2014.11.038

Ostojic, S. M., \& Stojanovic, M. D. (2014). Hydrogen-rich water affected blood alkalinity in physically active men. Research in Sports Medicine, 22(1), 49-60. https://doi.org/10.1080/15438627.2013.852092

Patwardhan, A. R., Vallurupalli, S., Evans, J. M., Bruce, E. N., \& Knapp, C. F. (1995). Override of spontaneous respiratory pattern generator reduces cardiovascular parasympathetic influence. Journal of Applied Physiology, 79(3), 1048-1054. https://doi.org/10.1152/iappl.1995.79.3.1048

Peçanha, T., Paula-Ribeiro, M., Campana-Rezende, E., Bartels, R., Marins, J. C. B., \& de Lima, J. R. P. (2014). Water intake accelerates parasympathetic reactivation after high-intensity exercise. International Journal of Sport Nutrition \& Exercise Metabolism, 24(5), 489-496. https://doi.org/10.1123/iisnem.2013-0122 Penttilä, J., Helminen, A., Jartti, T., Kuusela, T., Huikuri, H. V., Tulppo, M. P., Coffeng, R., \& Scheinin, H. (2001). Time domain, geometrical and frequency domain analysis of cardiac vagal outflow: Effects of various respiratory patterns. Clinical Physiology, 21(3), 365-376. https://doi.org/10.1046/i.1365-2281.2001.00337.x

Routledge, H. C., Chowdhary, S., Coote, J. H., \& Townend, J. N. (2002). Cardia vagal response to water ingestion in normal human subjects. Clinical Science, 103(2), 157-162. https://doi.org/10.1042/cs1030157

Schroeder, C., Bush, V. E., Norcliffe, L. J., Luft, F. C., Tank, J., Jordan, J., \& Hainsworth, R. (2002). Water drinking acutely improves orthostatic tolerance in healthy subjects. Circulation, 106(22), 2806-2811. https://doi.org/10.1161/01. cir.0000038921.64575.d0

Scott, E. M., Greenwood, J. P., Gilbey, S. G., Stoker, J. B., \& Mary, D. A. (2001). Water ingestion increases sympathetic vasoconstrictor discharge in normal human subjects. Clinical Science, 100(3), 335-342. https://doi.org/10.1042/ cs1000335

Shimouchi, A., Nose, K., Yamaguchi, M., Ishiguro, H., \& Kondo, T. (2009). Breath hydrogen produced by ingestion of commercial hydrogen water and milk. Biomarker Insights, 4, Article BMI-S2209. https://doi.org/10.4137/bmi.s2209

Steptoe, A., Hamer, M., \& Chida, Y. (2007). The effects of acute psychological stress on circulating inflammatory factors in humans: A review and meta-analysis. Brain, Behavior, and Immunity, 21(7), 901-912. https://doi.org/10.1016/i bbi.2007.03.011

Task Force of the European Society of Cardiology. (1996). Heart rate variability: Standards of measurement, physiological interpretation and clinical use. Circulation, 93(5), 1043-1065. https://doi.org/10.1161/01.cir.93.5.1043

Tomofuji, $T$, Kawabata, $Y$, Kasuyama, K., Endo, $Y$, Yoneda, T., Yamane, M. Azuma, T., Ekuni, D., \& Morita, M. (2014). Effects of hydrogen-rich water on aging periodontal tissues in rats. Scientific Reports, 4(1), Article 5534. https:// doi.org/10.1038/srep05534

Wang, H.-M., \& Huang, S.-C. (2012). SDNN/RMSSD as a surrogate for LF/HF: A revised investigation. Modelling and Simulation in Engineering, 2012, Article 931943. https://doi.org/10.1155/2012/931943

Zanini, D., Stajer, V., \& Ostojic, S. M. (2020). Hydrogen vs. caffeine for improved alertness in sleep-deprived humans. Neurophysiology, 52(1), 67-72. https://doi. org/10.1007/s11062-020-09852-7 